\title{
L 1 Generalized Procrustes 2D Shape Alignment
}

\section{Larsen, Rasmus}

\section{Published in:}

Journal of Mathematical Imaging and Vision

Link to article, DOI:

10.1007/s10851-008-0077-2

Publication date:

2008

Link back to DTU Orbit

\section{Citation (APA):}

Larsen, R. (2008). L 1 Generalized Procrustes 2D Shape Alignment. Journal of Mathematical Imaging and Vision, 31(2-3), 189-194. https://doi.org/10.1007/s10851-008-0077-2

\section{General rights}

Copyright and moral rights for the publications made accessible in the public portal are retained by the authors and/or other copyright owners and it is a condition of accessing publications that users recognise and abide by the legal requirements associated with these rights.

- Users may download and print one copy of any publication from the public portal for the purpose of private study or research.

- You may not further distribute the material or use it for any profit-making activity or commercial gain

- You may freely distribute the URL identifying the publication in the public portal

If you believe that this document breaches copyright please contact us providing details, and we will remove access to the work immediately and investigate your claim 


\title{
$L_{1}$ Generalized Procrustes 2D Shape Alignment
}

\author{
Rasmus Larsen \\ DTU Informatics, \\ Technical University of Denmark, \\ Richard Petersens Plads, Building 321, \\ DK-2800 Kgs. Lyngby rl@imm.dtu.dk
}

(Published in J Math Imaging Vis 82008) 31:189-194)

\begin{abstract}
This paper describes a new method for resistant and robust alignment of sets of $2 \mathrm{D}$ shapes wrt. position, rotation, and iso-tropical scaling. Apart from robustness a major advantage of the method is that it is formulated as a linear programming (LP) problem, thus enabling the use of well known and thoroughly tested standard numerical software. The problem is formulated as the minimization of the norm of a linear vector function with a contraint of non-zero size. This is achieved by using the city block distance between points in the plane. Unfortunately the city block distance is dependent on the orientation of the coordinate system, i.e. it is not rotationally invariant. However, by simultaneously minimizing the city block distances in a series of rotated coordinate systems we are able to approximate the circular equidistance curves of Euclidean distances with a regular polygonal equidistance curve to the precision needed. Using 3 coordinate systems rotated $30^{\circ}$ we get a 12 sided regular polygon, with which we achieve deviations from Euclidean distances less than $2 \%$ over all directions. This new formulation allows for minimization in the $L_{1}$-norm using LP. We demonstrate that the use of the $L_{1}$-norm results in resistance towards object as well as landmark outliers. Examples that illustrate the properties of the robust norm are given on simulated as well as a biological data sets.
\end{abstract}

\section{Introduction}

The study of the geometry of classes of objects represented by sets of (corresponding) landmark points is usually concerned with the variation up to a given transformation. The most common set of transformations applied is the Euclidean similarity transformation, i.e. translation, rotation, and iso-tropical scale. Using Kendall's (1) intuitive definition the shape of an object is the geometrical information that remains when effects due to Euclidean similarity transformations are filtered out. Analysis of the shape variability across a set 
of examples requires the alignment of the shapes to a common frame of reference. This is usually obtained by means of a generalized Procrustes analysis (GPA) $(2 ; 3 ; 4)$. The GPA consists of minimizing the sum of squared distances between corresponding landmarks on all examples and a reference shape with respect to the reference shape and similarity transformations of all example shapes, i.e. GPA is a least squares estimator.

Several problems may occur when aligning a set of shapes. Methods for extracting landmarks - be they manual, semi-automated, or fully automated may result in missing points on some shapes, landmark outliers, and even errors in the correspondence between landmarks. Object outliers may also be present.

Least squares methods are not particularly good at handling these situations. Other alignment procedures that handle these problems are therefore necessary. The insensitivity to outliers - landmark or object - is usually referred to as resistance, and insensitivity to correspondence errors, i.e. model breakdown, is called robustness (5).

Other work on robust and resistant alignment has been done. Siegel $(6 ; 7)$ used double repeated medians to achieve robust alignment in order to study the differences in shapes. Dryden and Walker (8) used S-estimators. In more general settings Huttenlocher (9) used Hausdorff distances between point sets, and Fischler and Bolles (10) based the alignment on consensus of random samplings of subsets of landmarks (i.e. the RANSAC procedure).

A standard way of achieving resistance is to avoid the quadratic penalty of least squares by applying a linear penalty. We will formulate the $2 \mathrm{D}$ alignment problem as the minimization of the norm of a linear vector function. This formulation is particular to $2 \mathrm{D}$ and does not extend to landmarks in 3 or more dimensions.

Applying the $L_{2}$-norm will result in the usual least squares 2D GPA. Using the $L_{1}$-norm will result in the minimization of the city block distance between corresponding landmarks of all examples and a reference shape. This minimization problem is formulated as a linear programming (LP) problem. However, the use of the city block distance obviously introduces dependence of the orientation of the chosen coordinate system. In order to achieve rotational independence we use a linear approximation to Euclidean distance based on averages of city block distances in equi-angularly rotated coordinate systems. In the Ph.D. thesis by Fikret Er (11) initial work on $L_{1}$-norm 2D GPA is done using the Expectation Maximization (12) algorithm. In (13) Ordinary $L_{1}$ Procrustes Analysis is shown to suffer from a non-uniqueness problem.

The main contribution in this work is the formulation of the $L_{1}$-norm $2 \mathrm{D}$ GPA as an LP problem. Thus allowing the use of standard, numerically stable, and well understood algorithms guaranteeing to find the global optimum.

\section{The alignment task}

Let there be given $L$ training examples for a given shape class, and let each example be represented by a configuration of $K \geq 32 \mathrm{D}$ landmark points not 
all coincident. Let these configurations be represented by complex vectors

$$
\mathbf{z}_{l}=\left(x_{l 1}+i y_{l 1}, \ldots, x_{l K}+i y_{l K}\right)^{T}, \quad l=1, \ldots, L .
$$

Assume the generative model (4)

$$
z_{l}=\frac{1}{a_{l}}\left(\mu+\epsilon_{l}\right)-b_{l},
$$

i.e. each shape is modelled as a pertubation $\left(\epsilon_{l} \in \mathbb{C}^{K}\right)$ of a modal shape $\boldsymbol{\mu}=\left(\mu_{1}, \ldots, \mu_{K}\right)^{T} \in \mathbb{C}^{K}$ - that subsequently undergoes a Euclidean similarity transformation. $a_{l} \in \mathbb{C}$ represents $2 \mathrm{D}$ translation and $b_{l} \in \mathbb{C}$ scale-rotation.

The alignment problem in 2D (2) consists of estimating the modal shape and the set of Euclidean similarity parameters for each shape so as to minimize the size of the residuals, $\boldsymbol{\epsilon}_{l}, l=1, \ldots, L$.. The modal shape can be recovered up to a Euclidean similarity transformation, and the procedure is called a generalized Procrustes analysis (GPA). The least squares solution to GPA consists of minimization of

$$
\mathrm{GPA}=\sum_{l=1}^{L} \sum_{k=1}^{K}\left|a_{l} z_{l k}+b_{l}-\mu_{k}\right|^{2},
$$

where the complex norm is given by $\left|z_{l k}\right|=\sqrt{x_{l k}^{2}+y_{l k}^{2}}$. In order to avoid the degenerate solution $a_{l}=b_{l}=0, l=1, \ldots, L$ and $\boldsymbol{\mu}=\mathbf{0}$ it is customary to require that the mean shape, $\boldsymbol{\mu}$ is centered and of unit size (5), i.e. $\bar{\mu}=\frac{1}{K} \sum_{k=1}^{K} \mu_{k}=0$ and size $S(\boldsymbol{\mu})=\left\|\boldsymbol{\mu}-\bar{\mu} \mathbf{1}_{K}\right\|=1$.

Let $\hat{a}_{l}$ and $\hat{b}_{l}$ be the estimates of pose parameters and $\hat{\boldsymbol{\mu}}_{0}$ the estimated mean shape resulting from minimization of GPA in Eq. (2). In some situations it is useful to fix the modal shape at the average scale-rotation $(\bar{a})$ and possibly translation $(\bar{b})$ of the set of sample shapes, i.e.

$$
\begin{aligned}
\hat{\boldsymbol{\mu}} & =\bar{a} \hat{\boldsymbol{\mu}}_{0}+\bar{b} \\
\bar{a} & =\frac{1}{L} \sum_{l=1}^{L} \hat{a}_{l} \\
\bar{b} & =\frac{1}{L} \sum_{l=1}^{L} \hat{b}_{l},
\end{aligned}
$$

Alternative 1. As an resistant alternative to using least squares we may choose to minimize the sum of absolute Euclidean lengths of the residuals, i.e. minimize

$$
\mathrm{RGPA}_{1}=\sum_{l=1}^{L} \sum_{k=1}^{K}\left|a_{l} z_{l k}+b_{l}-\mu_{k}\right|
$$

w.r.t. the modal shape and Euclidean similarity transformation parameters.

Alternative 2. As a second alternative we may choose to minimize the sum of city block lengths of the residuals, i.e. minimize

$$
\mathrm{RGPA}_{2}=\sum_{l=1}^{L} \sum_{k=1}^{K}\left|\operatorname{Re}\left\{a_{l} z_{l k}+b_{l}-\mu_{k}\right\}\right|+\left|\operatorname{Im}\left\{a_{l} z_{l k}+b_{l}-\mu_{k}\right\}\right|,
$$


where Re and Im denote real and imaginary part of a complex number, respectively.

\subsection{Formulation of the RPGA $\mathrm{R}_{2}$, minimization problems}

Using a multiple linear regression formulation as described in (4) the alignment problem - RPGA $\mathrm{R}_{2}$ - consists of the minimization of a vector function $\boldsymbol{F}$ w.r.t. the $L_{1}$ norm.

$$
\boldsymbol{F}=\left[\begin{array}{l}
\boldsymbol{\mu}-\boldsymbol{Z}_{1} \boldsymbol{\Theta}_{1} \\
\boldsymbol{\mu}-\boldsymbol{Z}_{2} \boldsymbol{\Theta}_{2} \\
\vdots \\
\boldsymbol{\mu}-\boldsymbol{Z}_{L} \boldsymbol{\Theta}_{L}
\end{array}\right] \text { where } \boldsymbol{Z}_{l}=\left[\begin{array}{cccc}
x_{l 1} & -y_{l 1} & 1 & 0 \\
\vdots & \vdots & \vdots & \vdots \\
x_{l K} & -y_{l K} & 1 & 0 \\
y_{l 1} & x_{l 1} & 0 & 1 \\
\vdots & \vdots & \vdots & \vdots \\
y_{l K} & x_{l K} & 0 & 1
\end{array}\right]
$$

w.r.t. $\boldsymbol{\Theta}_{l}=\left[\operatorname{Re}\left\{a_{l}\right\}, \operatorname{Im}\left\{a_{l}\right\}, \operatorname{Re}\left\{b_{l}\right\}, \operatorname{Im}\left\{b_{l}\right\}\right]^{T}, l=1, \ldots, L$ and $\boldsymbol{\mu}$. The $l$ th aligned shape is given by $\boldsymbol{Z}_{l} \boldsymbol{\Theta}_{l}$. Rewriting $\mathbf{F}$, we see that it is linear in the parameters, $\phi=\left[\boldsymbol{\Theta}_{1}, \boldsymbol{\Theta}_{2}, \ldots, \boldsymbol{\Theta}_{L}, \boldsymbol{\mu}\right]^{T}$,

$$
\mathbf{F}(\phi)=\left[\begin{array}{lllll}
\mathbf{Z}_{1} & \mathbf{0} & \ldots & \mathbf{0} & -\mathbf{I} \\
\mathbf{0} & \mathbf{Z}_{2} & \ldots & \mathbf{0} & -\mathbf{I} \\
\vdots & & \ddots & & \vdots \\
\mathbf{0} & \ldots & \ldots & \mathbf{Z}_{L} & -\mathbf{I}
\end{array}\right]\left[\begin{array}{l}
\Theta_{1} \\
\boldsymbol{\Theta}_{2} \\
\vdots \\
\boldsymbol{\Theta}_{L} \\
\boldsymbol{\mu}
\end{array}\right]
$$

An choice of parameters that minimizes any norm of $\mathbf{F}$ is to choose $\phi=\mathbf{0}$, that is, collapse everything to a point. The minimization needs to be constrained to not allow this degenerate solution. A set of restrictions - linear in the parameters - are

$$
\sum_{l=1}^{L} \operatorname{Re}\left\{a_{l}\right\}=c_{1} \quad \sum_{l=1}^{L} \operatorname{Im}\left\{a_{l}\right\}=c_{2} \quad \sum_{l=1}^{L} \operatorname{Re}\left\{b_{l}\right\}=c_{3} \quad \sum_{l=1}^{L} \operatorname{Im}\left\{b_{l}\right\}=c_{4}
$$

In our examples we will use $c_{1}=L, c_{2}=0$, and $c_{3}=c_{4}=0$, which means that the modal shape will be centered and have the average scale and rotation of all original objects.

\subsubsection{Formulating the LP problem}

The minimization of the norm of $F$ is equivalent to finding a vector $\mathbf{o}=$ $\left[\begin{array}{llll}o_{1} & O_{2} & \ldots & o_{2 K L}\end{array}\right]$ whose elements bound the values of the alignment function, $\boldsymbol{F}(\phi)$ of Eq. (5), that is $\left|F_{m}(\phi)\right| \leq o_{m}, m=1, \ldots, 2 K L$, or equivalently

$$
-o_{m} \leq F_{m}(\phi) \leq o_{m}
$$


such that the following criterion are minimized

$$
\mathrm{RGPA}_{2}: \quad \sum_{m} o_{m}
$$

The values of $o_{m}$ take on the role of the absolute values of the residuals in each coordinate. Expanding the indices Eq. (6) can be stated as

$$
\begin{aligned}
& -o_{x l k} \leq x_{l k} \operatorname{Re}\left\{a_{l}\right\}-y_{l k} \operatorname{Im}\left\{a_{l}\right\}+\operatorname{Re}\left\{b_{l}\right\}-\operatorname{Re}\left\{\mu_{k}\right\} \leq o_{x l k} \\
& -o_{y l k} \leq y_{l k} \operatorname{Re}\left\{a_{l}\right\}+x_{l k} \operatorname{Im}\left\{a_{l}\right\}+\operatorname{Im}\left\{b_{l}\right\}+\operatorname{Re}\left\{\mu_{k}\right\} \leq o_{y l k} .
\end{aligned}
$$

\subsubsection{Properties of the solutions}

The equidistance curve for the Manhattan distance applied in Eq. (4) are shown in Fig. 1(a) A strong directional dependency is obvious. In general this dependency may render the alignment of sets of shapes dependent on the choice of coordinate system. This is obviously undesirable. However, under certain models rotational invariance is none the less possible.

From the underlying GPA model in Eq. (1) we have

$$
\epsilon_{l k}=a_{l} z_{l k}+b_{l}-\mu_{k}
$$

If the distribution of the $\epsilon$ 's for fixed $l$ is circular symmetric then the a change of coordinate system will not change the optimum of the objective function for the RGPA 2 criterion.

Let us assume that we have determined the optimal parameters across all coordinate systems. For RGPA 2 at the optimum the coordinates of each $\mu_{k}$ will be the medians of the distribution of the residuals along the real and imaginary axis. If the distribution is circular symmetric these medians will not change as the coordinate system rotates. Therefore in this situation we will find the same optimum irrespectively of the choice of orientation of the coordinate system.

Moreover, an advantage of using the linear vector function formulation is that it is easy to deal with missing observations, that is, missing landmarks in shapes. This is done just by leaving out the rows from the coefficient matrix that correspond to the missing points.

\subsection{Formulation of the RPGA $A_{1}$ minimization problems}

If circular symmetry of the residuals cannot be assumed we can apply RGPA . Here rotational invariance is obtained by using the Euclidean metric, as is illustrated by its equi-distance curve in Fig. 1(b). However, this involves substituting the linear objective function Eq. (7) with a non-linear one.

$$
\sum_{i} \sum_{j} \sqrt{o_{x i j}^{2}+o_{y i j}^{2}}
$$

A linear approximation to the Euclidean equi-distance curve is obtained by taking the sum of Manhattan distances in a series of rotated coordinate 


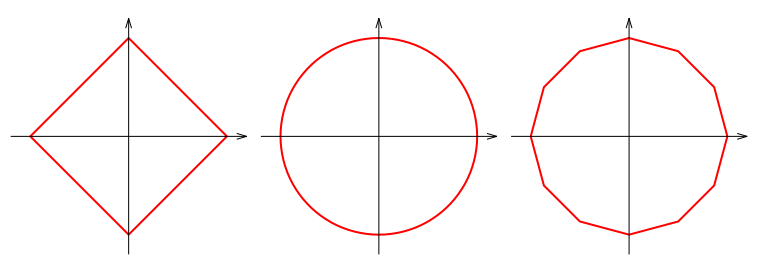

(a)

(b)

(c)

Figure 1: Equidistance curves for (a) the Manhattan, (b) the Euclidean, and (c) the 12 sided polygonal approximation metrics

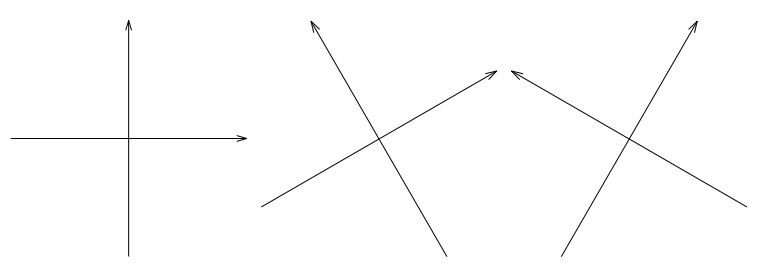

Figure 2: Coordinate systems used for approximating Euclidean distance. The systems are rotated $0, \pi / 6$, and $\pi / 3$, respectively.

systems. Using the 3 coordinate systems shown in Fig. 2 we achieve the equidistance curve shown in Fig. 1(c). The distance between a point $(x, y)$ in the plane and origo using this sum of Manhattan distances from $N$ equi-angularly rotated coordinate systems is

$$
\sum_{n=1}^{N}|x \cos (n \Delta \theta)-y \sin (n \Delta \theta)|+|y \cos (n \Delta \theta)+x \sin (n \Delta \theta)|, \quad \Delta \theta=\frac{\pi}{2 N}
$$

It is easily shown that

$$
\int_{0}^{\pi / 2}|x \cos \theta-y \sin \theta|+|y \cos \theta+x \sin \theta| d \theta \propto \sqrt{x^{2}+y^{2}} .
$$

Which means that after suitable normalization increasing $N$ will in the limit result in the desired Euclidean distance. Using the $N=3$ approximation we achieve deviations from the Euclidean equi-distance curve in the range $\pm 1.7 \%$.

\subsubsection{Properties of the solution}

The minimization of the criterion $\mathrm{RGPA}_{1}$ can be viewed as a generalization the resistant ordinary Procrustes analysis (OPA) defined in $(11 ; 13)$. OPA consists of aligning one set of landmarks, $\boldsymbol{z}_{1}$ to a fixed set, $\boldsymbol{z}_{2}$ using a Euclidean similarity transformation, i.e. minimize

$$
\mathrm{ROPA}=\sum_{k=1}^{K}\left|a_{l} z_{1 k}+b_{l}-z_{2 k}\right|
$$




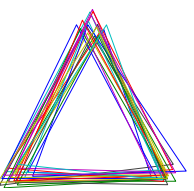

(a) unaligned

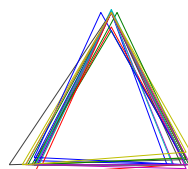

(b) $l_{1}$ norm

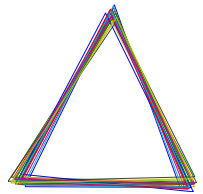

(c) $l_{2}$ norm

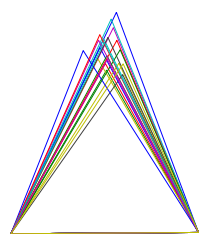

(d) $l_{1}$ norm

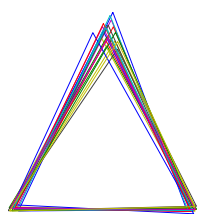

(e) $l_{2}$ norm

Figure 3: (a) 10 triangles. (b-c) alignment based on the 3 corner points using the $L_{1}$ and $L_{2}$, respectively. (d-e) aligned as (b-c) but with an additional 18 landmarks distributed equidistantly on the lower side included.

Er (11) developed an expectation maximization algorithm for the optimization, and Lima (13) showed that in general the ROPA does not have a unique minimum. Particularly, if $\boldsymbol{z}_{1}$ is an isosceles triangle a set of optimal solutions will lie along a line segment in parameter space.

If we examine the RGPA criteria for the $L=2$ case we find

$$
\operatorname{RGPA}_{1}=\sum_{i=1}^{K}\left|a_{2}\left(z_{1 k}+z_{2 k}\right)-2 b_{2}-z_{1 k}\right|
$$

Here we have used the contraints $b_{1}+b_{2}=0$ and $a_{1}+a_{2}=1$. Thus it is obvious that the RGPA criteria also will suffer from non-uniqueness in specific situations. In this particular case from the analogy to ROPA we see that it occurs when $\boldsymbol{z}_{1}+\boldsymbol{z}_{2}$ is an isosceles triangle.

\section{Examples}

\subsection{Triangles}

The choice of landmarks can have high influence on the result of the alignment of a set of shapes. This is illustrated in Fig. 3 where the alignment of triangles with different number of landmarks is compared. The triangles were generated by creating a reference triangle and then adding i.i.d. Gaussian noise to each corner. They were then aligned in the $L_{1}$ and $L_{2}$ norms. Then between the bottom two points of the triangles additional 18 points were added by linear interpolation. The triangles were then realigned. The extra 18 points are added to illustrate and study the influence of different annotations of the same shapes.

We see that the $L_{1}$ as well as the $L_{2}$ norm alignment are sensitive to the chosen representation, In the case of densely sampling the lower side the $L_{1}$ alignment regards the top corner point as an outlier and disregards it thereby achieving perfect alignment of the the lower sides. Applying the $L_{1}$ norm identifies a single landmark as the source of variation between the input shapes. 


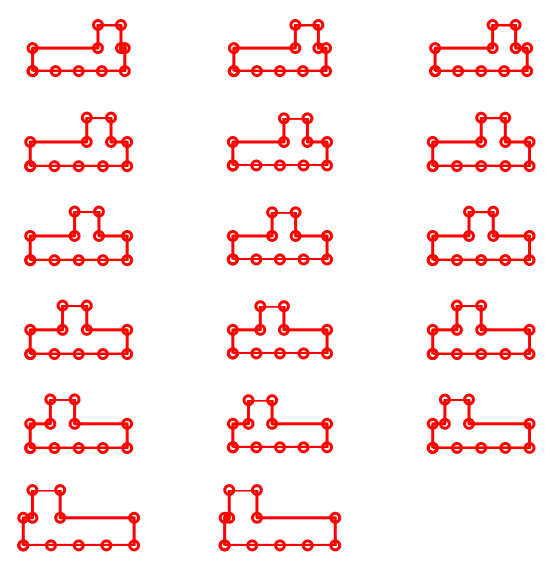

(a)

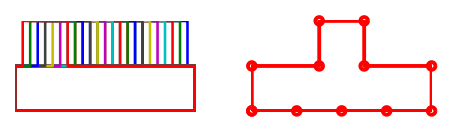

(b)

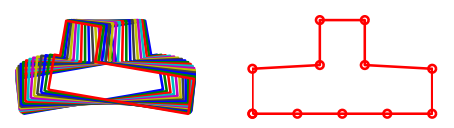

(c)

Figure 4: (a) 17 boxes with bumps in varying positions; boxes with bumps and their modal shape after (b) $L_{1}$-norm GPA, and (c) standard $L_{2}$-norm GPA. 


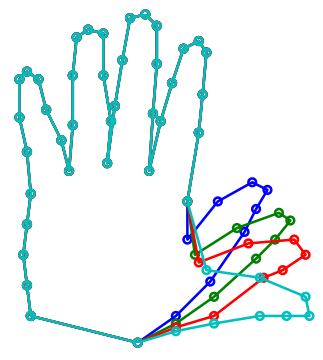

(a)

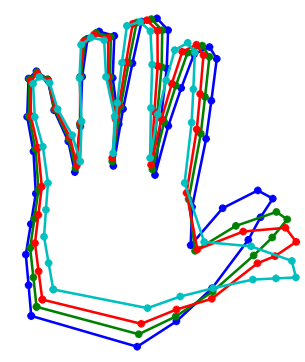

(b)

Figure 5: Four hands with animated thumb aligned using (a) $L_{1}$-norm GPA and (b) standard $L_{2}$-norm GPA.

\subsection{Boxes with bumps}

In the second examples we are considering a set of oblong boxes with a bump in varying position on top. In the initial configuration the boxes are perfectly aligned, cf. Fig. 4(a).

Now, applying $L_{1}$-norm alignment based on the approximated RGPA criterion yields exactly the initial configuration as desired, cf. Fig. 4(b). Applying standard GPA yields the alignment shown in Figs. 4(c).

The $L_{1}$ norm method has correctly identified the difference between the input shapes as the position of the bump, whereas standard GPA yields a solution that has introduced artificial scale and rotational variability. The modal shape form the $L_{2}$-norm experiment has slanting shoulders and is not representative of the class of shapes. Moreover, the boxes with the bumps in the most extreme positions are shrunk and rotated, relative to the others.

\subsection{Hands with animating thumb}

In a similar example a hand is recorded while moving the thumb. Again $L_{1}$-norm alignment (cf. Fig. 5(a)) correctly identifies that the input shapes only vary in terms of thumb orientation, whereas the $L_{2}$ GPA (cf. Fig. 5(b)) introduces scale and rotational variation not present in the input data.

\section{Conclusion}

The alignment of shapes into a common reference frame is a fundamental task in shape modeling. A natural extension to the general Procrustes alignment usually employed is to use other norms than the $L_{2}$ norm. Here the $L_{1}$ norm has been explored. 
A general Procrustes analysis based on absolute Euclidean distances yields a non-linear optimization problem. However, using a linear approximation based on averaging the Manhattan distance of a series of equi-angularly rotated coordinate systems we can achieve as near an approximation as desired. Furthermore, under certain constraints general Procrustes analysis based on the Manhattan distance gives the same result as a general Procrustes analysis based on absolute Euclidean distances. In both cases the optimization problem can be formulated as a linear programming problem. This allows the usage of standard, well understood numerical methods such as linear programming. Lots of research has been done in the field of linear programming and the properties of the algorithms used to solve linear programming tasks, such as convergence, are well understood. The coefficient matrix is very structured and sparse. That can be exploited when implementing the algorithms to speed up the alignment process.

Examples illustrating the properties of the method are provided - both artificial and real. An important property is that the resulting alignments form $L_{1}$-norm based general Procrustes methods yields solutions that are sparse in terms of the input (or landmarks).

\section{Acknowledgements}

The author wishes to thank Associate Professor, Ph.D. Thomas Stidsen, DTU Management, Technical University of Denmark with good help and advice on linear programming.

\section{References}

[1] D. G. Kendall, "The diffusion of shape," Advances in Applied Probability, vol. 9, pp. 428-430, 1977.

[2] J. C. Gower, "Generalized Procrustes analysis," Psychometrika, vol. 40, pp. 33-50, 1975.

[3] J. M. F. ten Berge, "Orthogonal Procrustes rotation for two or more matrices," Psychometrika, vol. 42, pp. 267-276, 1977.

[4] C. Goodall, "Procrustes methods in the statistical analysis of shape," Journal of the Royal Statistical Society, Series B, vol. 53, no. 2, pp. 285-339, 1991.

[5] I. L. Dryden and K. Mardia, Statistical Shape Analysis. Chichester: John Wiley \& Sons, 1998. xx +347 pp.

[6] A. F. Siegel, "A robust comparison of biological shapes," Biometrics, vol. 38, pp. 341-350, June 1982.

[7] A. F. Siegel, "Robust regression using repeated medians," Biometrika, vol. 69 , no. 1 , pp. $242-244,1982$. 
[8] I. L. Dryden and G. Walker, "Highly resistant regression and object matching," Biometrics, vol. 55, pp. 820-825, Sept. 1999.

[9] D. P. Huttenlocher, G. A. Klanderman, and W. J. Rucklidge, "Comparing images using the Hausdorff distance," IEEE Transactions on Pattern Analysis and Machine Intelligence, vol. 15, no. 9, pp. 850-863, 1993.

[10] M. A. Fischler and R. C. Bolles, "Random sample consensus: A paradigm for model fitting with applications to image analysis and automated cartography," Communications of the ACM, vol. 24, no. 6, pp. 381-395, 1981.

[11] F. Er, Robust methods in Statistical Shape Analysis. PhD thesis, University of Leeds, Department of Statistics, Leeds, UK, 1998. x + 86 pp.

[12] A. Dempster, N. Laird, and D. Rubin, "Maximum likelihood from incomplete data via the EM algorithm," Journal of the Royal Statistical Society, Series B, 1977.

[13] V. M. C. Lima and J. T. Kent, "The non-uniqueness problem in $l_{1}$ ordinary Procrustes analysis," Student, vol. 4, Feb. 2003. 